\title{
Room-temperature photoluminescence of $\mathrm{BaTiO}_{3}$ : Joint experimental and theoretical study
}

\author{
E. Orhan* and J. A. Varela \\ Instituto de Química, Universidade Estadual Paulista, 14801-907 Araraquara, SP, Brazil
}

A. Zenatti

Interunidades-USP-Departamento de Química, Universidade Federal de São Carlos, 13565-905 São Carlos, SP, Brazil

M. F. C. Gurgel, F. M. Pontes, E. R. Leite, and E. Longo

Departamento de Química, Universidade Federal de São Carlos, 13565-905 São Carlos, SP, Brazil

P. S Pizani

Departamento de Física, Universidade Federal de São Carlos, 13565-905 São Carlos, SP, Brazil

A. Beltràn and J. Andrès

Departament de Ciències Experimentals, Universitat Jaume I, P.O. Box 6029 AP, 12080 Castelló, Spain

(Received 15 July 2004; revised manuscript received 25 August 2004; published 15 February 2005)

\begin{abstract}
Strong photoluminescent emission has been measured at room temperature for noncrystalline $\mathrm{BaTiO}_{3}(\mathrm{BT})$ perovskite powders. A joint experimental and theoretical study has been carried out to rationalize this phenomenon. From the experimental side, BT powder samples have been synthesized following a soft chemical processing, their crystal structure has been confirmed by x-ray data and the corresponding photoluminescence (PL) properties have been measured. Only the structurally disordered samples present PL at room temperature. From the theoretical side, first-principles quantum-mechanical techniques, based on density-functional theory at the B3LYP level, have been employed to study the electronic structure of crystalline (BT-c) and asymmetric (BT-a) models. Theoretical and experimental results are found to be consistent and their confrontation leads to an interpretation of the PL apparition at room temperature in the structurally disordered powders.
\end{abstract}

DOI: 10.1103/PhysRevB.71.085113

PACS number(s): 71.15.Mb, 71.20.-b

\section{INTRODUCTION}

The development of semiconductor materials with active optical properties such as photoluminescence (PL), electroluminescence, or nonlinear optic properties may lead to new optoelectronic devices with superior performance. Much interest has been paid in the study of PL in structurally disordered or nanostructured materials since the first time visible PL at room temperature was observed in porous silicon. ${ }^{1}$ The $\mathrm{PL}$ at low temperatures of crystalline $\mathrm{ABO}_{3}$ perovskite has long been known and recent studies attest for the current interest in those semiconducting compounds, ${ }^{2-4}$ owing to their wide variety of unique electronic, magnetic, and optical properties. ${ }^{5,6}$ Much attention has also been paid on $\mathrm{BaTiO}_{3}$ (BT) crystal samples, particularly since it was discovered that, when pure BT crystal samples are excited by radiation above their energy band gap, a broad visible luminescent band appears at low temperatures $(45 \mathrm{~K}))^{7,8}$ In nanocrystalline BT, PL has also been detected at room temperature and with excitation energies lower than the energy band gap. This PL has been attributed to the size of the particles and to the presence of oxygen vacancies in the lattice. ${ }^{2,9,10}$ Our group reported intense visible PL at room temperature in $\mathrm{ATiO}_{3}$ powders and thin-film compounds (where $A=\mathrm{Pb}, \mathrm{Ca}$, $\mathrm{Sr}$, and $\mathrm{Ba}$ ), independently of the size of the grains, but depending on the rate of crystalline disorder existing in the compounds, opening up an interesting field of research for this class of materials. ${ }^{11-14}$

In this paper, we present measurements of broad PL band at room temperature as a function of heat treatment of
$\mathrm{BaTiO}_{3}$ powder prepared by the polymeric precursor method. ${ }^{15,16}$ This process offers advantages over other synthesis techniques such as low cost, good compositional homogeneity, high purity, low processing temperatures, and the ability to coat large substrate areas. The origin of the PL is investigated in terms of electronic structure calculations realized in the framework of first-principles quantummechanical techniques. The aim of this synergetic strategy between experimental results and electronic structure study is not to explain how PL occurs, as many valuable hypotheses already exist, ${ }^{17-19}$ but to explain why it occurs at room temperature in the noncrystalline powders.

The layout of the paper is as follows: In Sec. II, the experimental methods of the powder preparation are described, together with spectral measurement techniques. Section III presents the computational details, while Sec. IV gives the crystal structure and periodic models used for the calculations. The results are discussed attempting to explain the PL phenomenon in Sec. V. At last, Sec. VI resumes the main lines of our hypothesis of the origin of the strong PL at room temperature in structurally disordered BT powders.

\section{EXPERIMENT}

$\mathrm{BaTiO}_{3}(\mathrm{BT})$ powders studied in the present work were derived from a soft chemical processing, the polymeric precursors method. In this method, titanium citrates were formed by the dissolution of titanium isopropoxide in an 
aqueous solution of citric acid $\left(60-70{ }^{\circ} \mathrm{C}\right)$. After homogenization of the Ti solution, $\mathrm{BaCO}_{3}$ was slowly added. In order to achieve total $\mathrm{BaCO}_{3}$ dissolution, ammonium hydroxide was added drop by drop until the $p \mathrm{H}$ reached 7-8. The complete dissolution of the salts produced a clear solution. After complete dissolution of the $\mathrm{BaCO}_{3}$ salt, ethylene glycol was added to promote polymerization of the mixed citrates by polyesterification reaction. The molar ratio between the barium and titanium cations was $1: 1$ and the citric acid/ ethylene glycol ratio was fixed at 60/40 (mass ratio). The heat treatment was carried out at different temperatures for $20 \mathrm{~h}$, at a heating rate of $10^{\circ} \mathrm{C} / \mathrm{min}$, in a tube furnace in an oxygen flow to promote pre-pyrolysis and total oxidation of the organic precursor. The BT powders were structurally characterized using x-ray diffraction (XRD) $(\mathrm{Cu} K \alpha$ radiation). The diffraction patterns were recorded on a Siemens D5000 machine in a $\theta-2 \theta$ configuration, using a graphite monochromator. Two kinds of powders have to be distinguished for a better understanding of this work, the first one, whose thermal treatment was realized below the crystallization temperature, and the other one, whose crystallization has been reached. The spectral dependence of optical absorbance for the crystalline and still disordered powders was measured at room temperature in the total reflection mode, using Cary 5G equipment. The PL spectra were taken at room temperature with a U1000 Jobin-Yvon double monochromator coupled to a cooled GaAs photomultiplier and a conventional photon counting system. The 488.0 exciting wavelength of an argon ion laser was used, with the laser's maximum output power kept at $20 \mathrm{mw}$. A cylindrical lens was used to prevent the sample from overheating. The slit width used was $100 \mathrm{~mm}$.

\section{COMPUTATIONAL METHODS}

Calculations have been carried out with the CRYSTAL98 ${ }^{20}$ package within the framework of the density-functional theory using the gradient-corrected correlation functional by Lee, Yang, and Parr, combined with the Becke3 exchange functional, B3LYP, ${ }^{21,22}$ which has been demonstrated by Muscat et ll $^{23}$ to be suitable for calculating structural parameters and band structures for a wide variety of solids. The atomic centers have been described by all electron basis sets 9763-311(d631)G for $\mathrm{Ba}^{24}{ }^{24}$ 86-411(d31)G for Ti, and 6-31G* for $\mathrm{O}^{25}$ The $k$-points sampling was chosen to be 40 points within the irreducible part of the Brillouin zone. For simulating the displacement of the $\mathrm{Ti}$ atom, as explained in the next section, we have used the ATOMDISP option provided with the CRYSTAL program.

\section{CRYSTAL STRUCTURE AND PERIODIC MODELS}

BT crystallizes in the tetragonal perovskite structure (space group $P 4 / \mathrm{mmm}$ ). The barium atoms share the corners of the unit cell and the titanium is at the center, surrounded by six oxygens that occupy the middle of the faces, in an elongated octahedral configuration. We have used a $1 \times 1$ $\times 2$ supercell as a periodic model for representing the $\mathrm{BT}$ crystalline phase (BT-c). It results in ten atoms in the unit
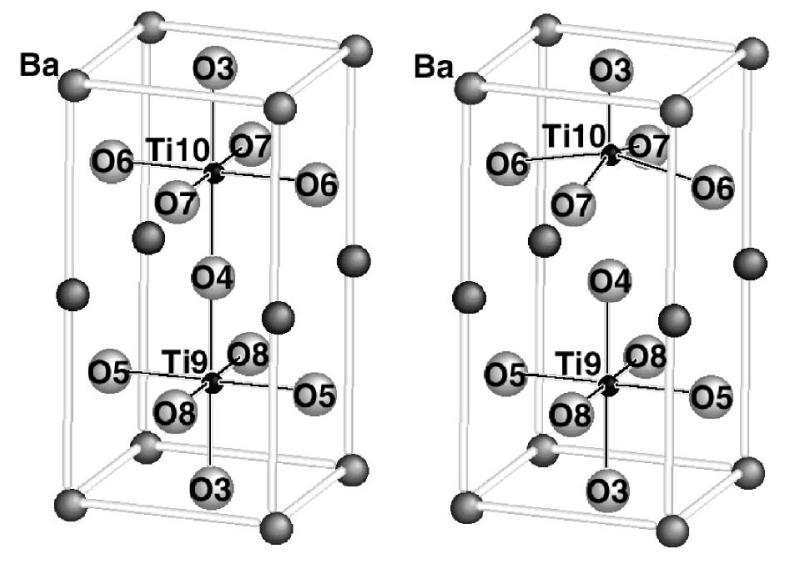

FIG. 1. (Left) BT-c periodic model: $1 \times 1 \times 2$ supercell of the $\mathrm{BaTiO}_{3}$ unit cell. (Right) BT-a periodic model: Ti10 was shifted by a (l) 00.5$)$ - $\AA$ vector.

cell, see Fig. 1. This BT-c model can be designed as $\left[\mathrm{TiO}_{6}\right]$ $\left[\mathrm{TiO}_{6}\right]$, as each titanium is surrounded by six oxygen ions in a slightly distorted $O h$ configuration. The experimental values of a and c parameters are 3.99 and $4.04 \AA$, respectively, and the calculated ones 3.970 and $4.006 \AA$.

$\mathrm{X}$-ray-absorption fine-structure (XAFS) measurements performed on BT characterized an ordered-disordered structure where the ions suffer off-center displacements. ${ }^{26} \mathrm{X}$-ray appearance near-edge structure (XANES) experimental results on $\mathrm{SrTiO}_{3}$ and $\mathrm{PbTiO}_{3}$ phases that had been synthesized by the polymeric precursor method and whose thermal treatment had been done at temperatures inferior to their crystallization threshold pointed out the coexistence of two types of environments for the titanium, namely, fivefold coordination $\left(\left[\mathrm{TiO}_{5}\right]\right.$ square-base pyramid $)$ and sixfold $\left(\left[\mathrm{TiO}_{6}\right]\right.$ octahedron). ${ }^{13,14}$ This certain degree of order in structurally disordered materials was to be expected, since two or more atoms arranged close to each other in a stable configuration must necessarily have some degree of order because there always are minima of the potential energy. Furthermore, as it is well known, the details of the band structure for a periodic system is mainly determined by the potential within the unit cell, rather than by the long-range periodicity. This means that any symmetry perturbation in the unit cell will have consequences on the electronic structure. Yacobi et al. ${ }^{26}$ also demonstrated that a model containing local disordered distortions in the high-symmetry mode accounts quantitatively for both displacivelike and order-disorder-like properties. Based on these experimental results, we modeled the BT structurally disordered phases by shifting Ti10 by a $\left(\begin{array}{lll}0 & 0 & 0.5\end{array}\right)-\AA$ vector from its previous position in the former $1 \times 1 \times 2$ supercell. This displacement asymmetries the unit cell, in which Ti10 is now surrounded by five oxygens in a squarebase pyramid configuration while Ti9 surroundings remains six oxygens as in the BT-c case. This Ti10 displacement is the simplest way to represent the two experimentally detected environment of $\mathrm{Ti},\left[\mathrm{TiO}_{5}\right]$ (square-base pyramid) and $\left[\mathrm{TiO}_{6}\right]$ octahedron. Therefore this asymmetric BT model, $\mathrm{BT}-\mathrm{a}$, represents the BT structurally disordered material. This structure can be designed as $\left[\mathrm{TiO}_{6}\right]-\left[\mathrm{TiO}_{5}\right]$, see Fig. 1. Our aim with this modelization is to offer a simple scheme allow- 


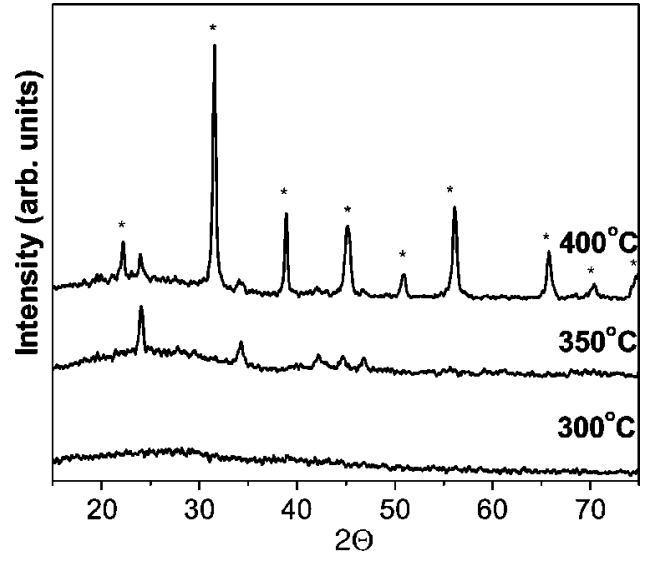

FIG. 2. XRD patterns for three powder samples heat treated at 300,350 , and $400{ }^{\circ} \mathrm{C}$ for $20 \mathrm{~h}$ in an oxygen flow. The $*$ mark the $\mathrm{BaTiO}_{3}$ phase.

ing us to understand the effects of structural deformation on the electronic structure without completely removing the geometry of the cell that is useful for the periodic calculation. We are aware that it does not represent the exact structure of disordered $\mathrm{BaTiO}_{3}$, which does not present any order at medium and long range. We are focusing on the effect of one local structural deformation among the numerous possible irregularities. This kind of model has already been successfully employed for studying $\mathrm{PL}$ in disordered $(\mathrm{Ba}, \mathrm{Sr}) \mathrm{TiO}_{3}$, $\mathrm{Pb}(\mathrm{Zr}, \mathrm{Ti}) \mathrm{O}_{3}$, and $\mathrm{SrTiO}_{3} \cdot{ }^{27-29}$

\section{RESULTS AND DISCUSSION}

Figure 2 shows the $\mathrm{x}$-ray-diffraction patterns of three BT powder samples annealed in an oxygen flow at 300,350 , and $400{ }^{\circ} \mathrm{C}$ for $20 \mathrm{~h}$. A diffuse XRD pattern can be observed for the sample heat treated at $300{ }^{\circ} \mathrm{C}$, indicating the formation of an inorganic precursor, in which crystallization has not yet occurred after the pyrolysis process. At $350{ }^{\circ} \mathrm{C}$, the powder presents some diffraction peaks belonging to the well-known oxycarbonates that commonly appear during the synthesis of $\mathrm{BT}$ in those conditions. ${ }^{30}$ Moreover, the shape of the pattern clearly indicates that a part of the powder is not yet crystallized. The BT crystalline phase can be observed from $400{ }^{\circ} \mathrm{C}$ and is marked by *. No intermediate titanate phase was observed, suggesting that a direct crystallization occurred from the structurally disordered phase. Some oxycarbonate peaks yet can be observed but is has been proved that their presence does not influence the PL results. ${ }^{31}$

Figure 3 shows the photoluminescence spectra, at room temperature, of the same three samples. The spectra general aspect is a broad band covering a large part of the visible spectra. Those bands can be decomposed into three components: three broad bands in the green, orange, and red regions. At $350{ }^{\circ} \mathrm{C}$, the $\mathrm{PL}$ intensity is at its maximum, the orange and green contributions being the most important. For the sample heat treated at $350{ }^{\circ} \mathrm{C}$, the PL intensity strongly decreases. The three bands are shifted towards the higher wavelength and orange and red components, of lower energy, become the most important. From $400{ }^{\circ} \mathrm{C}$, the powders are

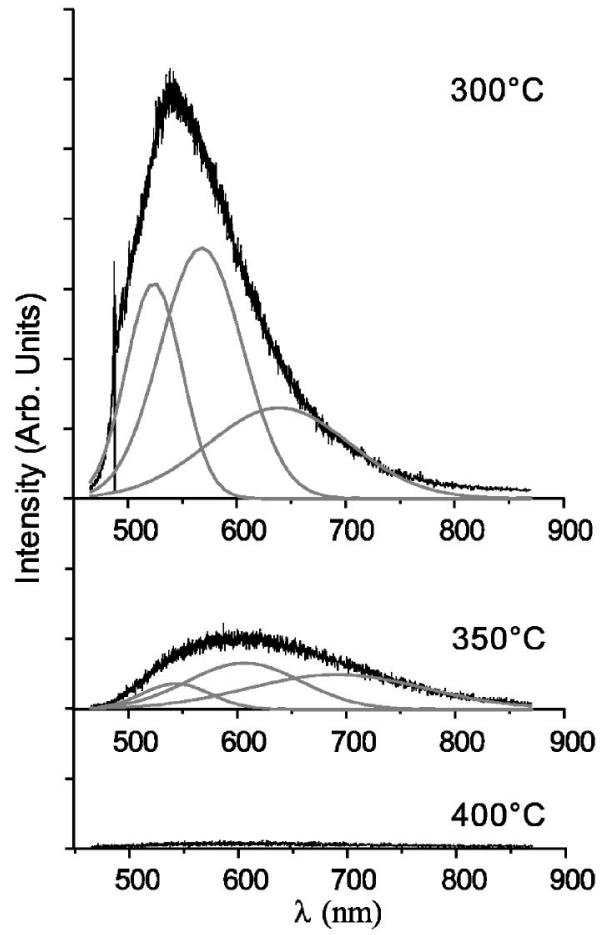

FIG. 3. Room-temperature photoluminescence spectra of $\mathrm{BaTiO}_{3}$ powder samples annealed at 300,350 , and $400{ }^{\circ} \mathrm{C}$ for $20 \mathrm{~h}$ in an oxygen flow. The exciting wavelength was the 488-nm line of an argon ion laser. The gray curves are the curves resulting from the decomposition of the PL spectra.

well crystallized and their PL vanishes. The PL intensity is thus linked to the thermal treatment history of the powders, and thus to the structural disorder. The three components of the PL emission bands are probably linked to specific atomic arrangements. Interestingly, it can be noted that the PL emission does not depend on the network modifier because in the case of $(\mathrm{Ba}, \mathrm{Sr}) \mathrm{TiO}_{3}$ thin films heat treated at $300{ }^{\circ} \mathrm{C}$, the emission band maximum is also located around $550 \mathrm{~nm} .{ }^{27}$ The structural transformations occurring from disordered to ordered phases starts from the early stage of the polyesterification of the citrate solution containing the titanium and barium ions. The titanium who is the lattice former tends ideally to bond with six oxygens, but before it reaches this ideal configuration, there exist various coordination numbers for Ti in the structure. When the crystallization is reached, only the $\mathrm{TiO}_{6}$ cluster exists and the PL vanishes, showing that a complete order is not suitable for a good PL emission. Before the crystallization, the structure is a mixture of $\mathrm{TiO}_{x}$ clusters and in the majority, as we know from our XANES studies, $\mathrm{TiO}_{5}$ and $\mathrm{TiO}_{6}$ clusters, intercalated by $\mathrm{Ba}$ atoms. ${ }^{13,14}$ The higher the heat treatment temperature, the rarer the $\mathrm{TiO}_{5}$ conformation and the more ordered the structure. The PL spectra show that a complete disorder is not favorable to a high PL emission, as the spectrum of the $300{ }^{\circ} \mathrm{C}$ sample is much higher than the one of the $350{ }^{\circ} \mathrm{C}$ sample.

When the structurally disordered powders are excited by various wavelength laser lines, it also presents a strong PL with a linear dependence between the maximum emission 


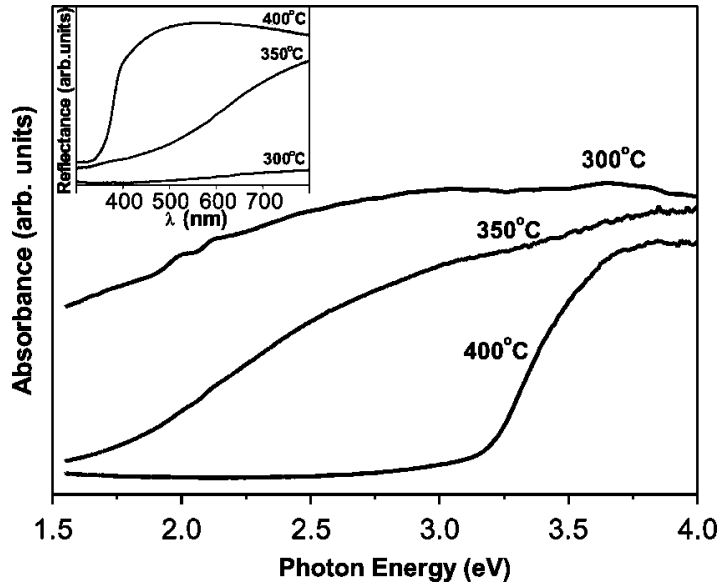

FIG. 4. Spectral dependence of the absorbance for the $\mathrm{BaTiO}_{3}$ powder samples annealed at 300,350 , and $400{ }^{\circ} \mathrm{C}$ for $20 \mathrm{~h}$ in an oxygen flow.

peak wavelength and the exciting wavelength, ${ }^{11}$ characteristic of radiative recombination of self-trapped excitons. ${ }^{32}$ This result suggests that the emission can be tuned by the exciting wavelength, thus that the PL of the structurally disordered powders occurs from a continuous excitation process.

In Fig. 4, the spectral dependence of the absorbance for the three BT samples are presented. The crystalline powder presents one well-defined absorption front while the structurally disordered powders exhibit typically a continuous smooth absorption increase as a function of the energy, suggesting the presence of localized states inside the band gap. The optical gaps obtained by extrapolation of the linear curve regions according to the Wood and Tauc method ${ }^{33}$ are $3.10 \mathrm{eV}$ for the crystalline powder and respectively 0.84 and $1.60 \mathrm{eV}$ for the disordered 300 and $350{ }^{\circ} \mathrm{C}$ annealed powders. The absorbance measurements, associated with the PL characterization of disordered BT powder, suggest a nonuniform band-gap structure with a tail of localized states and mobile edges.

As explained by Blasse and Grabmaier, ${ }^{17}$ the PL arises from a radiative return to the ground state, a phenomenon

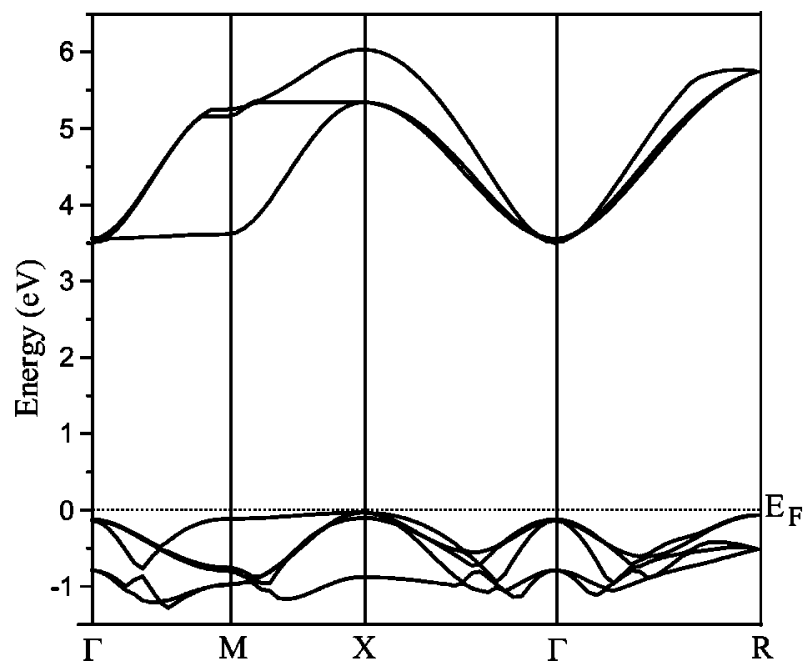

that is in concurrence with the nonradiative return to the ground state where the energy of the excited state is used to excite the vibrations of the host lattice. The radiative emission process occurs more easily if it exists in the structure trapped holes or trapped electrons. In order to evidence the presence of trapped holes and trapped electrons in our disordered powders, we performed a detailed theoretical study of the electronic structure in a crystalline, BT-c, and disordered (asymmetric) model, BT-a. To analyze the differences in the electronic structure, it is convenient to make reference to quantities such as the band gap or the projected densities of states (DOS) which may be compared to each other independently of the crystalline space group.

The left part of Fig. 5 reports the calculated band structure of bulk BT-c. The top of the valence band (VB) is at the $X$ point and is very close to the $\Gamma$ point. The bottom of conduction band $(\mathrm{CB})$ is at $\Gamma$. The minimal indirect gap between $X$ and $\Gamma$ is $3.53 \mathrm{eV}$, close to the experimental one deduced from the observed optical-absorption edge, that we found to be $3.10 \mathrm{eV}$ (Fig. 4). The minimal direct gap at $\Gamma$ is $3.66 \mathrm{eV}$. The calculated band structure of bulk BT-a is depicted in the right part of Fig. 5. The top of the VB is at the $X$ point and the bottom of CB is at $\Gamma$, as in the case of BT-c. The indirect minimal gap between $X$ and $\Gamma$ is $2.37 \mathrm{eV}$ while the minimal direct gap at $\Gamma$ is $2.47 \mathrm{eV}$. These results show that our data are consistent with the interpretation that the band gap is controlled by the degree of disorder, structural and thermal, in the lattice of BT system. The calculated total and atomresolved projected DOS of BT-c and BT-a models are shown in Fig. 6, ranging from $-5 \mathrm{eV}$ below the top of the $\mathrm{VB}$ to 8 $\mathrm{eV}$ above. In the case of BT-c, the upper $\mathrm{VB}$ is predominately made of the $\mathrm{O}(2 p)$ states, equivalently distributed on the six oxygens of the structure (left part of Fig. 6). In the case of BT-a (right part of Fig. 6), although the VB is also made of the $\mathrm{O}(2 p)$ states, the upper part, i.e., the new states, present a strong $\mathrm{O} 4$ character, the oxygen atom that loses the connection with Ti10 (Fig. 1). The CB is clearly made of the $\mathrm{Ti}(3 d)$ states in both cases. The Ti-O covalent bond creates a limited $\mathrm{Ti}(3 d)$ contribution in the $\mathrm{O}(2 p)$ region as well as a weak $\mathrm{O}(2 p)$ contribution to the $\mathrm{Ti}(3 d)$ area. The $\mathrm{Ba}(6 s)$

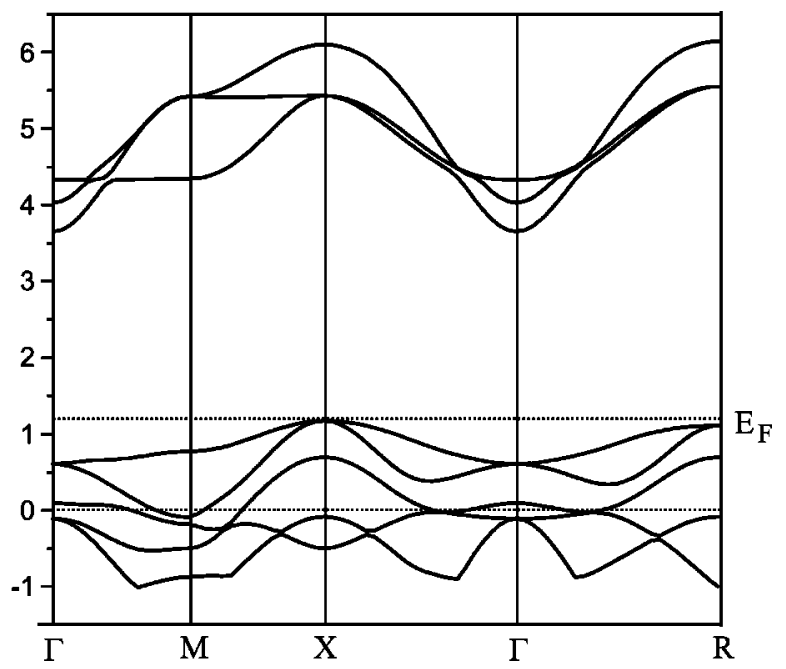

FIG. 5. Band structure for the BT-c (left) and BT-a (right) models. In both cases, the zero corresponds to the Fermi energy of the BT-c structure. 

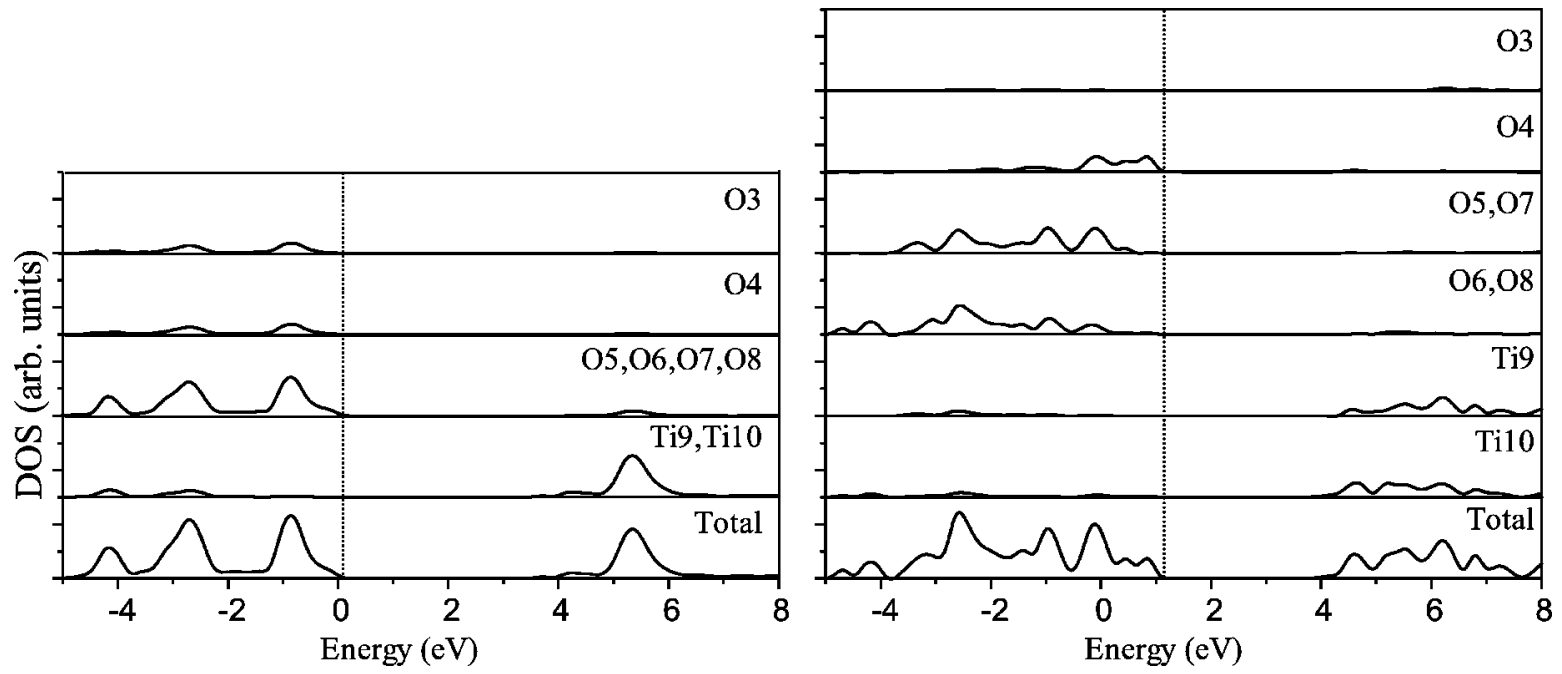

FIG. 6. Total and atom-projected density of states for the BT-c model (left) and for the BT-a model (right).

states are found between -10.5 and $-11.5 \mathrm{eV}$ in the case of BT-c and, more dispersed, between -9.5 and $-10.5 \mathrm{eV}$ in the case of BT-a. These levels are weakly hybridized with the oxygen levels.

It is also interesting to complement the present results with the analysis of the contour and surface plots of the electronic charge density calculated on a selected plane for BT-c and BT-a structures (Fig. 7 top and bottom, respectively). The chosen plane is a vertical diagonal plane containing the $\mathrm{Ba} 1, \mathrm{Ba} 2, \mathrm{Ti}$, Ti10, O3, and $\mathrm{O} 4$ atoms. It is shown by the gray areas in the cell representations. An analysis of Fig. 7 clearly shows that the bonding between $\mathrm{Ba}$ and $\left[\mathrm{TiO}_{6}\right]$ is strongly ionic, while a covalent bonding nature is visible between $\mathrm{Ti}$ and $\mathrm{O}$, a result of the hybridization between the
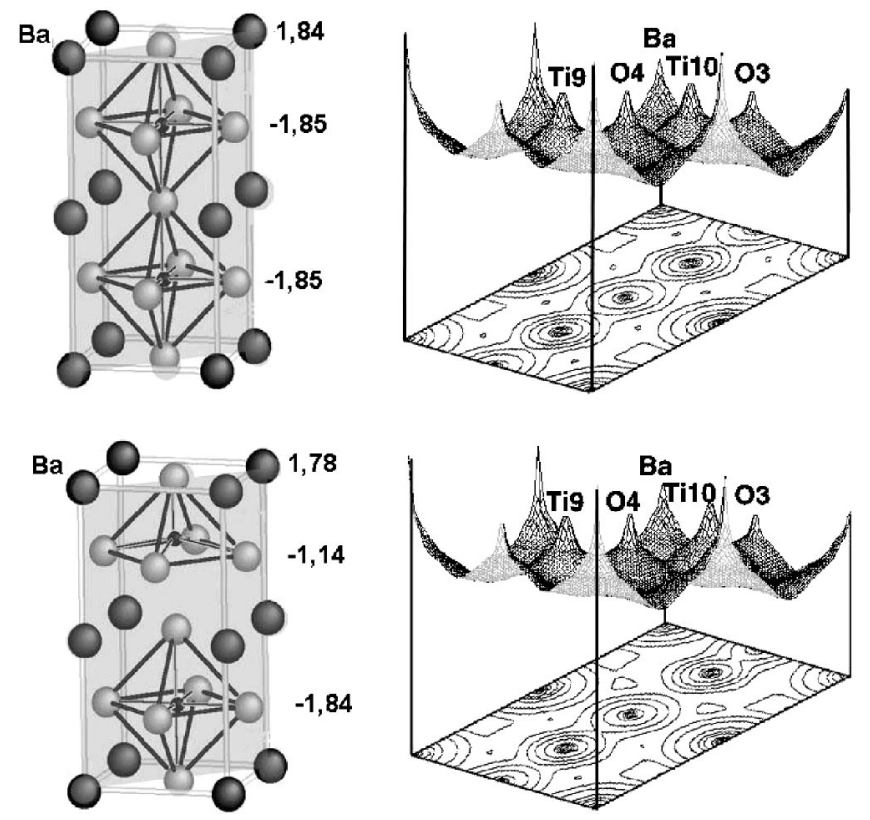

FIG. 7. The charge-density contour and surface plots for BT-c and BT-a models in a vertical diagonal plane represented by the gray area. The numbers are the formal charges of Ba network and $\left[\mathrm{TiO}_{6}\right] /\left[\mathrm{TiO}_{5}\right]$ clusters.
$\mathrm{O}(2 p)$ and the $\mathrm{Ti}(3 d)$ states. The breaking of the Ti10-O4 bond is visible in the bottom of Fig. 7. The displacement of a metallic center on going from BT-c to BT-a results in the deformation of a symmetric $\left[\mathrm{TiO}_{6}-\mathrm{TiO}_{6}\right]$ structure into two fragments: $\left[\mathrm{TiO}_{6}\right]$ and $\left[\mathrm{TiO}_{5}\right]$. The formal charges indicated on the crystal cell in the left margin of Fig. 7 show that, as expected, the symmetric $\left[\mathrm{TiO}_{6}-\mathrm{TiO}_{6}\right]$ possesses a regular charge repartition on both $\left[\mathrm{TiO}_{6}\right]$ clusters, $-1.85|e|$ and $3.68|e|(2 \times 1.84|e|)$ on the complete $\mathrm{Ba}$ network. In the case of the deformed structure, the formal charges of the $\left[\mathrm{TiO}_{6}\right]$ and $\left[\mathrm{TiO}_{5}\right]$ clusters become respectively $-1.14|e|$ and $-1.84|e|$, and the Ba network charge remains roughly the same, $3.42|e|$. A permanent charge gradient is thus created between $\left[\mathrm{TiO}_{6}\right]$ and $\left[\mathrm{TiO}_{5}\right]$ to compensate the breaking of the Ti10-O4 bond (as defined in Fig. 1). However, the net atomic charges of $\mathrm{Ti}$ and $\mathrm{O}$ centers individually suffer few changes: Ti9 gains $0.02|e|$, Ti10 $0.06|e|$, and all the $\mathrm{O}$ less than $-0.1|e|(\mathrm{O} 4-0.068|e|)$. Therefore the negative charge transfer occurs from one cluster to another and not from the oxygen atom to one titanium atom, as widely seen in the literature.

Figure 8 presents the results of a calculation in a $\mathrm{BaTiO}_{3}$ tetragonal unit cell in terms of the energy diagram. The evolution from the slightly distorted $\left[\mathrm{TiO}_{6}\right]$ octahedron to the

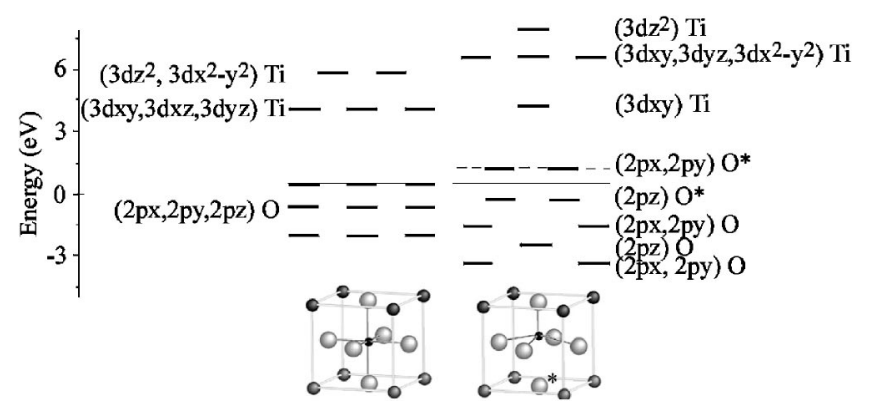

FIG. 8. Energy diagram of the frontier crystalline orbitals (CO's) taken at $\Gamma$ point for two simple tetragonal perovskite unit cells: a regular cell (left) and a cell where the Ti atom was shifted

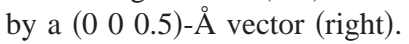


$\left[\mathrm{TiO}_{5}\right]$ square base pyramid entity of the crystal orbitals (CO's) whose main characters are $\mathrm{Ti} 3 d$ and $\mathrm{O} 2 p$ atomic orbitals is presented. The left diagram shows that the $\mathrm{Ti}(3 d)$ CO's of the conduction band present a " 2 g-eg" splitting classical for an octahedral ligand field. When the distortion occurs through the displacement of the Ti atom, there is a degeneracy lift, exactly like in the case of a Jahn-Teller interaction. Therefore our distorted model BT-a can also be seen as a crystalline model where Jahn-Teller distortion would have occurred every two $\left[\mathrm{TiO}_{6}\right]$ clusters. The charge transfer occurring from one $\left[\mathrm{TiO}_{5}\right]$ to one $\left[\mathrm{TiO}_{6}\right]$ cluster creates electron and hole polarons that can be designed as JahnTeller bipolarons. They have already been evidenced by means of electron paramagnetic resonance measurements in pure $\mathrm{BaTiO}_{3}$ monocrystal ${ }^{34}$ or by theoretical calculations where they were considered as point defects. ${ }^{35,36}$ Stashans et al. announced that they had to be expected for perovskitetype titanates. ${ }^{37}$ However, all the previous studies locate the hole polarons on the oxygen atom and the electron polaron on the titanium atom. The present work shows that this widely spread off concept may be erroneous since the $\left[\mathrm{TiO}_{x}\right]$ $(x=5,6)$ cluster entities are the centers of charge transfer. Those polarons, that already exist in the ground state, facilitate the emission process leading to PL, i.e., the radiative recombination. Our disordered BT powders thus intrinsically possess the necessary condition for creating PL, which is not the case for the crystalline powder where the quasiperfect structure does not allow the creation of point defects.

\section{CONCLUSIONS}

Powders of $\mathrm{BaTiO}_{3}$ have been synthesized following a soft chemical processing. They were structurally characterized by means of x-ray diffraction. The corresponding PL properties have been measured and only the structurally dis- ordered samples present the property. To understand the mechanism of this PL behavior in disordered $\mathrm{BaTiO}_{3}$, we turned to first-principles theory as an appropriate tool of rationalization. Therefore we have performed quantummechanical calculations on two models that stand for the crystalline and structurally disordered $\mathrm{BaTiO}_{3}$ powders. The electronic structures are examined in terms of band diagrams, density of states, electronic charges, and energy diagram. The theoretical results are confronted with experimental data and are in good agreement. The theoretical results indicate that the formation of a fivefold oxygen titanium coordination $\left[\mathrm{TiO}_{5}\right]$ through the displacement of Ti10 and the rupture of the Ti10-O4 bond (as defined in Fig. 1), introduces localized electronic levels with $\mathrm{O} 4(2 p x, 2 p y, 2 p z)$ character above the VB of the structure before deformation. The crystalline model presents indeed a higher band gap than the disordered model, which is in agreement with experimental gap determined from absorbance spectral data. Moreover, a charge transfer occurs from the $\left[\mathrm{TiO}_{5}\right]$ cluster to the $\left[\mathrm{TiO}_{6}\right]$ one, revealing the intrinsic presence of trapped holes and electrons, Jahn-Teller bipolarons, in the disordered phase of BT powders. The presence of those trapped holes and electrons, existing before the excitation, is the main factor allowing that the radiative emission process strongly prevails on the nonradiative recombination process in disordered perovskite compounds.

\section{ACKNOWLEDGMENTS}

This work was partially supported by the Brazilian research-financing institutions Fundação de Amparo à Pesquisa do Estado de São Paulo-FAPESP/CEPID and Conselho Nacional de Desenvolvimento Científico e Tecnológico-CNPq/PRONEX, and by the Programa de Colaboración Hispano-Brasileño No. PHB2001-0040.
*Fax: +55 $16 \quad 3351 \quad 8350$; electronic address: emmanuelle.orhan@liec.ufscar.br

${ }^{1}$ L. T. Canham, Appl. Phys. Lett. 57, 1046 (1990).

${ }^{2}$ J. Meng, Y. Huang, W. Zhang, Z. Du, Z. Zhu, and G. Zou, Phys. Lett. A 205, 72 (1995).

${ }^{3}$ H. Rinnert, M. Vergat, G. Marchal, and A. Burneau, Appl. Phys. Lett. 72, 3157 (1998).

${ }^{4}$ J. F. Scott, Ferroelectr. Rev. 1, 1 (1998).

${ }^{5}$ A. J. Millis, Nature (London) 392, 147 (1998).

${ }^{6}$ B. W. Wessels, Annu. Rev. Mater. Sci. 25, 525 (1995).

${ }^{7}$ C. N. Berglund and H. J. Braun, Phys. Rev. 164, 790 (1967).

${ }^{8}$ V. Trepakov, V. Vikhnin, S. Eden, S. Kapphan, H. Hesse, J. Seglins, and L. Jastrabik, J. Korean J. Korean Phys. Soc. 32, 1113 (1998).

${ }^{9}$ J. Yu, J. Sun, J. Chu, and D. Tang, Appl. Phys. Lett. 77, 2807 (2000).

${ }^{10}$ M.-S. Zhang, Z. Yin, Q. Chen, W. Zhang, and W. Chen, Solid State Commun. 119, 659 (2001).

${ }^{11}$ E. R. Leite, F. M. L. Pontes, E. J. H. Lee, R. Aguiar, E. Longo, D. S. L. Pontes, M. S. J. Nunes, H. R. Macedo, P. S. Pizani, F.
Lanciotti, Jr., T. M. Boschi, J. A. Varela, and C. A. Paskocimas, Appl. Phys. A: Mater. Sci. Process. 74, 529 (2002).

${ }^{12}$ E. R. Leite, L. P. S. Santos, N. L. V. Carreno, C. A. Paskocimas, F. Lanciotti, Jr., P. S. Pizani, C. E. M. Campos, J. A. Varela, and E. Longo, Appl. Phys. Lett. 78, 2148 (2001).

${ }^{13}$ E. R. Leite, F. M. L. Pontes, E. C. Paris, C. A. Paskocimas, E. J. H. Lee, E. Longo, P. S. Pizani, J. A. Varela, and V. Mastelaro, Adv. Mater. Opt. Electron. 10, 235 (2000).

${ }^{14}$ F. M. Pontes, E. Longo, E. R. Leite, E. J. H. Lee, J. A. Varela, P. S. Pizani, C. E. M. Campos, F. Lanciotti, Jr., V. Mastellaro, and C. D. Pinheiro, Mater. Chem. Phys. 77, 598 (2002).

${ }^{15}$ F. M. Pontes, E. Longo, J. H. Rangel, M. I. Bernardi, E. R. Leite, and J. A. Varela, Mater. Lett. 43, 249 (2000).

${ }^{16}$ F. M. Pontes, J. H. G. Rangel, E. R. Leite, E. Longo, J. A. Varela, E. B. Araujo, and J. A. Eiras, Thin Solid Films 366, 232 (2000).

${ }^{17}$ G. Blasse and B. C. Grabmaier, Luminescent Materials (SpringerVerlag, Berlin, 1994).

${ }^{18}$ R. Leonelli and J. L. Brebner, Phys. Rev. B 33, 8649 (1986).

${ }^{19}$ R. I. Eglitis, E. A. Kotomim, and G. Borstel, Eur. Phys. J. B 27, 483 (2002). 
${ }^{20}$ V. R. Saunders, R. Dovesi, C. Roetti, M. Causa, N. M. Harrison, R. Orlando, and C. M. Zicovich-Wilson, CRYSTAL98 User's Manual (University of Torino, Torino, 1998).

${ }^{21}$ C. Lee, W. Yang, and R. G. Parr, Phys. Rev. B 37, 785 (1988).

${ }^{22}$ A. D. Becke, J. Chem. Phys. 98, 5648 (1993).

${ }^{23}$ J. Muscat, A. Wander, and N. M. Harrison, Chem. Phys. Lett. 342, 397 (2001).

${ }^{24}$ Available from http://www.tcm.phy.cam.ac.uk/mdt26/crystal.html

${ }^{25}$ Available from http://www.chimifm.unito.it/teorica/crystal/ crystal.html

${ }^{26}$ Y. Yacoby and Y. Girshberg, in Fundamental Physics of Ferroelectrics 2000, edited by R. E. Cohen, AIP Conf. Proc. No. 535 (AIP, Melville, NY, 2000), p. 56.

${ }^{27}$ E. Longo, E. Orhan, F. M. Pontes, C. D. Pinheiro, E. R. Leite, J. A. Varela, P. S. Pizani, T. M. Boschi, F. Lanciotti, Jr., A. Beltrn, and J. Andrs, Phys. Rev. B 69, 125115 (2004).

${ }^{28}$ M. S. Silva, M. Cilence, E. Orhan, M. S. Ges, M. A. C. Machado, L. P. S. Santos, C. O. Paiva-Santos, E. Longo, J. A. Varela, M. A. Zaghete, and P. S. Pizani, J. Lumin. (to be published).
${ }^{29}$ E. Orhan, F. M. Pontes, C. D. Pinheiro, E. R. Leite, T. M Boschi, P. S. Pizani, A. Beltrn, J. Andrs, J. A. Varela, and E. Longo, J. Solid State Chem. (to be published).

${ }^{30}$ P. Duran, F. Capel, D. Gutierrez, J. Tartaj, M. A. Banares, and C. Moure, J. Mater. Chem. 11, 1828 (2001).

${ }^{31}$ F. M. Pontes, C. D. Pinheiro, E. Longo, E. R. Leite, S. R. De Lazaro, R. Magnani, P. S. Pizani, T. M. Boschi, and F. Lanciotti, Jr., J. Lumin. 104, 175 (2003).

${ }^{32}$ G. G. Siu, X. L. Wu, Y. Gu, and X. M. Bao, Appl. Phys. Lett. 74, 1812 (1999).

${ }^{33}$ D. L. Wood and J. Tauc, Phys. Rev. B 5, 3144 (1972).

${ }^{34}$ S. Lenjer, O. F. Schirmer, H. Hesse, and Th. W. Kool, Phys. Rev. B 66, 165106 (2002).

${ }^{35}$ R. I. Eglitis, E. A. Kotomin, and G. Borstel, J. Phys.: Condens. Matter 14, 3735 (2002).

${ }^{36}$ H. Pinto and A. Stashans, Phys. Rev. B 65, 134304 (2002).

${ }^{37}$ A. Stashans. H. Pinto, and P. Sanchez, J. Low Temp. Phys. 130, 3/4 (2003). 